\title{
Heavy Quarkonium Potential and inclusive decay widths in terms of Wilson loops
}

\author{
Antonio Pineda ${ }^{\mathrm{a}}$ \\ ${ }^{a}$ Dept. d'Estructura i Constituents de la Matèria and IFAE, U. Barcelona \\ Diagonal 647, E-08028 Barcelona, Catalonia, Spain
}

We briefly summarize some results obtained by potential NRQCD in the non perturbative regime: 1) a systematic procedure to obtain the heavy quarkonium potential in the $1 / m$ expansion (with explicit expressions up to $O\left(1 / m^{2}\right)$ in terms of Wilson loops), and 2) a description of the heavy quarkonium inclusive decays to light particles, and of the NRQCD matrix elements (also the octet ones), in terms of the wave-function at the origen and some gluonic field-strength correlators.

Heavy quarkonium $(b-\bar{b}, c-\bar{c})$ systems have been traditionally described by potential models in the past, being their inverse size assumed to be of $O\left(\Lambda_{Q C D}\right)$ (and that $\Lambda_{Q C D} \ll m$, being $m$ the mass of the heavy quark). Potential models are characterized by the introduction of a, more or less, phenomenological potential in a Schrödinger equation. By assuming some functionality in $r$ and by fitting the free parameters of the potential, a good description of the heavy quarkonium spectrum was obtained. Nevertheless, there were two issues: 1) under which circumstances, and how, a pure Schrödinger formulation will emerge from QCD in the non-perturbative regime and, if so, 2 ) how to obtain the potentials from QCD, or, at least, how to relate them with objects eventually computable in QCD (then any potential model should, at least, be consistent with points 1) and $2)$ ). The use of effective field theories has helped to clarify when point 1) is satisfied and how it can be derived from QCD (see [1-3]). The resulting Lagrangian of the effective theory (that we name potential NRQCD (pNRQCD)) reads

$\mathcal{L}_{\mathrm{pNRQCD}}=S^{\dagger}\left(i \partial_{0}-h\left(\mathbf{x}_{1}, \mathbf{x}_{2}, \mathbf{p}_{1}, \mathbf{p}_{2}\right)\right) S$,

where $h$ is the Hamiltonian of the singlet, i.e. of the heavy quarkonium. Actually $h$ is only a function of $\mathbf{r}=\mathbf{x}_{1}-\mathbf{x}_{2}, \mathbf{p}_{1}, \mathbf{p}_{2}$, which is analytic in the two last operators but contains non-analyticities in $\mathbf{r}$. $\mathbf{p}_{1}=-i \boldsymbol{\nabla}_{\mathbf{x}_{1}}$ and $\mathbf{p}_{2}=-i \boldsymbol{\nabla}_{\mathbf{x}_{2}} . h$ has the following expansion up to order $1 / m^{2}$ :

$$
\begin{aligned}
& h\left(\mathbf{x}_{1}, \mathbf{x}_{2}, \mathbf{p}_{1}, \mathbf{p}_{2}\right)=\frac{\mathbf{p}_{1}^{2}}{2 m_{1}}+\frac{\mathbf{p}_{2}^{2}}{2 m_{2}}+V^{(0)} \\
& +\frac{V^{(1,0)}}{m_{1}}+\frac{V^{(0,1)}}{m_{2}}+\frac{V^{(2,0)}}{m_{1}^{2}}+\frac{V^{(0,2)}}{m_{2}^{2}}+\frac{V^{(1,1)}}{m_{1} m_{2}} .
\end{aligned}
$$

For the $V^{(2,0)}$ and $V^{(0,2)}$ potentials we define

$V^{(2,0)}=V_{S D}^{(2,0)}+V_{S I}^{(2,0)}, V^{(0,2)}=V_{S D}^{(0,2)}+V_{S I}^{(0,2)} \cdot(3)$

The spin-independent terms can be written as

$V_{S I}^{(2,0)}=\left\{\frac{\mathbf{p}_{1}^{2}}{2}, V_{\mathbf{p}^{2}}^{(2,0)}(r)\right\}+\frac{V_{\mathbf{L}^{2}}^{(2,0)}(r)}{r^{2}} \mathbf{L}_{1}^{2}+V_{r}^{(2,0)}(r)$,

and

$V_{S I}^{(0,2)}=\left\{\frac{\mathbf{p}_{2}^{2}}{2}, V_{\mathbf{p}^{2}}^{(0,2)}(r)\right\}+\frac{V_{\mathbf{L}^{2}}^{(0,2)}(r)}{r^{2}} \mathbf{L}_{2}^{2}+V_{r}^{(0,2)}(r),(5)$

where $\mathbf{L}_{1} \equiv \mathbf{r} \times \mathbf{p}_{1}$ and $\mathbf{L}_{2} \equiv \mathbf{r} \times \mathbf{p}_{2}$ and

$$
\begin{aligned}
& V_{\mathbf{p}^{2}}^{(2,0)}(r)=V_{\mathbf{p}^{2}}^{(0,2)}(r), V_{\mathbf{L}^{2}}^{(2,0)}(r)=V_{\mathbf{L}^{2}}^{(0,2)}(r), \\
& V_{r}^{(2,0)}(r)=V_{r}^{(0,2)}\left(r ; m_{2} \leftrightarrow m_{1}\right) .
\end{aligned}
$$

The spin-dependent part of $V^{(2,0)}$ is of the type

$V_{S D}^{(2,0)}=V_{L S}^{(2,0)}(r) \mathbf{L}_{1} \cdot \mathbf{S}_{1}$.

Analogously, for the $V^{(0,2)}$ potential we can write

$V_{S D}^{(0,2)}=-V_{L S}^{(0,2)}(r) \mathbf{L}_{2} \cdot \mathbf{S}_{2}$,

where $V_{L S}^{(2,0)}(r)=V_{L S}^{(0,2)}\left(r ; m_{2} \leftrightarrow m_{1}\right)$.

For the $V^{(1,1)}$ potential we define

$V^{(1,1)}=V_{S D}^{(1,1)}+V_{S I}^{(1,1)}$. 
The spin-independent part can be written as

$$
\begin{aligned}
& V_{S I}^{(1,1)}=-\frac{1}{2}\left\{\mathbf{p}_{1} \cdot \mathbf{p}_{2}, V_{\mathbf{p}^{2}}^{(1,1)}(r)\right\} \\
& -\frac{V_{\mathbf{L}^{2}}^{(1,1)}(r)}{2 r^{2}}\left(\mathbf{L}_{1} \cdot \mathbf{L}_{2}+\mathbf{L}_{2} \cdot \mathbf{L}_{1}\right)+V_{r}^{(1,1)}(r),
\end{aligned}
$$

while the spin-dependent part contains the following operators:

$$
\begin{aligned}
& V_{S D}^{(1,1)}=V_{L_{1} S_{2}}^{(1,1)}(r) \mathbf{L}_{1} \cdot \mathbf{S}_{2}-V_{L_{2} S_{1}}^{(1,1)}(r) \mathbf{L}_{2} \cdot \mathbf{S}_{1} \\
& +V_{S^{2}}^{(1,1)}(r) \mathbf{S}_{1} \cdot \mathbf{S}_{2}+V_{\mathbf{S}_{12}}^{(1,1)}(r) \mathbf{S}_{12}(\hat{\mathbf{r}}),
\end{aligned}
$$

where $\mathbf{S}_{12}(\hat{\mathbf{r}}) \equiv 3 \hat{\mathbf{r}} \cdot \boldsymbol{\sigma}_{1} \hat{\mathbf{r}} \cdot \boldsymbol{\sigma}_{2}-\boldsymbol{\sigma}_{1} \cdot \boldsymbol{\sigma}_{2}$ and $V_{L_{1} S_{2}}^{(1,1)}(r)=V_{L_{2} S_{1}}^{(1,1)}\left(r ; m_{1} \leftrightarrow m_{2}\right)$.

Point 2), i.e. how to obtain the relation of the potential with objects computable within QCD, can be summarized in the following question:

How to obtain the potential in terms of Wilson loops in the $1 / m$ expansion (also usually named adiabatic or Born-Oppenheimer approximation)?

The first attempts to answer this question started more than twenty years ago. The expression for the leading spin-independent potential, of $O\left(1 / m^{0}\right)$, corresponds to the static Wilson loop and was derived by Wilson and Susskind [4]:

$V^{(0)}(r)=\lim _{T \rightarrow \infty} \frac{i}{T} \ln \left\langle W_{\square}\right\rangle$.

Expressions for the leading spin-dependent potentials in the $1 / m$ expansion, of $O\left(1 / m^{2}\right)$, were given in Refs. [5]. The procedure followed in these works proved to be very difficult to extend beyond these leading-order potentials. In Ref. [6], a new method to calculate the potentials was proposed, where new spin-independent (some of them momentum-dependent) potentials at $O\left(1 / \mathrm{m}^{2}\right)$ were obtained. In [7], expressions for the spin-dependent potentials were obtained in terms of eigenstates of the static limit of the NRQCD Hamiltonian in the Coulomb gauge. In these works, the potentials did not correctly reproduce the ultraviolet behaviour expected from perturbative QCD (the hard $\operatorname{logs} \sim \log m$ ). This was the first signal that a controlled derivation of the potentials from QCD was needed. The solution to this problem needs of NRQCD [8] where the ultraviolet behavior is encoded in the matching coefficients of the NRQCD operators. It is then possible to incorporate them to the potentials as done in [9]. At that point, the obtained set of potentials at $O\left(1 / \mathrm{m}^{2}\right)$ seemed to be complete.

Nevertheless, this view has recently been challenged in Refs. [2,3], where a systematic study of the potential has been done within an effective field theory framework: pNRQCD. The main improvements achieved in Refs. [2,3] with respect these previous computations can be summarized as follows:

A) We have developed a general procedure to compute the potential by equating green functions in NRQCD and pNRQCD [2].

B) We have developed the general method and formally provide recursive equations to obtain the potential at any order in $1 / \mathrm{m}$ in terms of matrix elements and energies of the states solution of the static limit $[2,3]$. These expressions can then be rewritten in terms of Wilson loops.

Points A) and B) solve, in alternative ways, question 2) and, thus, finally settle this issue, opened since more than twenty years ago.

Once the formalism has been developed, we have been able to obtain, for the first time, the complete potential (up to field redefinitions) in pure gluedynamics up to $O(1 / m)$ in [2] and up to $O\left(1 / m^{2}\right)$ in [3]. If compared with the previous expressions for the potential that one may find in the literature, our novel findings can be summarized as follows:

C) A new $O(1 / m)$ potential [2].

D) Correct expressions for the spin/velocityindependent terms of the $1 / \mathrm{m}^{2}$ potential: $V_{r}^{(0,2)}\left(V_{r}^{(2,0)}\right)$ and $V_{r}^{(1,1)}[3]$.

E) We have clarified that the, so far, standard expression obtained by Eichten and Feinberg for $V_{L_{1} S_{2}}^{(1,1)}\left(V_{L_{2} S_{1}}^{(1,1)}\right)$ and later confirmed by several groups was incorrect [3].

Let us stress that, to date, A) and B) are the only available methods in the literature to com- 
pute the potential in terms of Wilson loops within a systematic expansion in $1 / m$. The attempts to implement the method of Eichten and Feinberg beyond their leading-order results were not able to obtain finite expressions [10]. Indeed, in a way, the procedure A) can be seen as the generalization of the Eichten and Feinberg method. In order to obtain this generalization it was crucial to understand the computation within an effective field theory ideology where equalities between Green functions were imposed and interpolating fields with arbitrary normalizations used. The method advocated in Ref. [6] does not appear to be correct, at least in its current formulation, since, for instance, it is not able to obtain the $1 / m$ potential. The computations in Ref. [7] essentially provide the correct expressions for the spin-dependent potentials (once one takes the NRQCD matching coefficients to tree level and neglects the tree-level annihilation contribution in the equal mass case). Nevertheless, their methodology needs to be generalized (along the lines of $[2,3]$ ) to take into account the fact that one is dealing with operators instead that with numbers in these type of computations.

Let us also notice that the expressions for the potential in terms of Wilson loops encode the full perturbative series in $\alpha_{s}$, at leading order in the multipole expansion, in the perturbative situation, which is a well defined limit.

Once $h$ has been obtained, we can obtain the energies of the bound states. At the order of interest, we take the energies from the real part of the Schrödinger equation

$(\operatorname{Re} h)\langle\mathbf{r} \mid n, l, s, j\rangle=E_{n j l s}\langle\mathbf{r} \mid n, l, s, j\rangle$,

with quantum numbers $n, j, l$ and $s$ as defined in Ref. [11]. From the imaginary piece of $h$, we can obtain the inclusive decay widths (to light hadrons, leptons or photons) by using the relation

$\Gamma=-2\langle n, l, s, j|\operatorname{Im} h| n, l, s, j\rangle$.

This is what we have done in Refs. [12,11] on which we report in what follows.

The imaginary parts of $h$ are inherited from the four-fermion matching coefficients of NRQCD: $\operatorname{Im} c_{4-f}^{d=6}, \operatorname{Im} c_{4-f}^{d=8}, \ldots$ Therefore, the structure of the imaginary part of the Hamiltonian reads

$$
\begin{aligned}
& \operatorname{Im} h \sim \sum_{k} \operatorname{Im} c_{4-f, k}^{d=6} \frac{\delta^{(3)}(\mathbf{r})}{m^{2}}\left(A_{k}+B_{k} \frac{\Lambda_{Q C D}^{2}}{m^{2}}+\ldots\right) \\
& +\sum_{k} \operatorname{Im} c_{4-f, k}^{d=8}\left(A_{k}^{\prime} \frac{\left\{\delta^{(3)}(\mathbf{r}), \nabla^{2}\right\}}{m^{4}}\right. \\
& \left.\quad+B_{k}^{\prime} \frac{\delta^{(3)}(\mathbf{x})}{m^{2}} \frac{\Lambda_{Q C D}^{2}}{m^{2}}+\ldots\right) \\
& +\sum_{k} \operatorname{Im} c_{4-f, k}^{d=8}\left(C_{k} \mathcal{T}_{S J}^{i j} \frac{\nabla^{i} \delta^{(3)}(\mathbf{r}) \boldsymbol{\nabla}^{j}}{m^{4}}+\ldots\right)+\ldots,
\end{aligned}
$$

being dictated by local potentials. The S-wave and $\mathrm{P}$-wave inclusive decay width easily follow

$$
\begin{aligned}
& \Gamma_{S-\text { wave }} \sim \sum_{k} \operatorname{Im} c_{4-f, k}^{d=6} \frac{\left|R_{n s 0 s}(0)\right|^{2}}{m^{2}} \\
& \times\left(A_{k}+B_{k} \frac{\Lambda_{Q C D}^{2}}{m^{2}}+\cdots\right) \\
&+\sum_{k} \operatorname{Im} c_{4-f, k}^{d=8}\left(A_{k}^{\prime} \frac{R_{n s 0 s}(0)\left(\nabla^{2} R_{n 10 s}(0)\right)}{m^{4}}\right. \\
&\left.+B_{k}^{\prime} \frac{\left|R_{n s 0 s}(0)\right|^{2}}{m^{2}} \frac{\Lambda_{Q C D}^{2}}{m^{2}}+\ldots\right)+\ldots, \\
& \Gamma_{P-\text { wave }} \sim \sum_{k} \operatorname{Im} c_{4-f, k}^{d=8} C_{k} \frac{\left|\nabla R_{n j 1 s}(0)\right|^{2}}{m^{4}}+\ldots,(16)
\end{aligned}
$$

where $R_{n s 0 s}$ and $R_{n j 1 s}$ are the $S$-wave and $P$ wave radial component of $\langle\mathbf{r} \mid n, l, s, j\rangle$.

Equations. (15-16) illustrate the factorization of the soft and ultrasoft scale since the coefficients $A_{k}, B_{k} \Lambda_{Q C D}^{2}, C_{k}$, etc. are independent of the bound-state dynamics. This makes possible to obtain model independent predictions from the above results, which just come out from the structure of our effective theory and the fact that the imaginary pieces are encoded in the four-fermion matching coefficients. In any case we are able to go further and to obtain the coefficients $A_{k}$, $B_{k} \Lambda_{Q C D}^{2}, C_{k}$, etc. in terms of gluonic correlators or the identity (a specific case of gluonic correlator). Therefore we can relate them with QCD. These correlators could be eventually computed in the lattice, evaluated with models or fixed with the experiment. For illustration, we show the non-perturbative parameter which appears in $P$ wave decays [12]: 
$C_{k} \sim \frac{1}{N_{c}} \int_{0}^{\infty} d t t^{3}\langle g \mathbf{E}(t) \cdot g \mathbf{E}(0)\rangle$.

The explicit expressions for the gluonic correlators can be found in Refs. [12,11], where Eqs. (15-16) have been obtained with relative precision $O\left(\Lambda_{Q C D}^{2} / m^{2}\right)$.

The traditional description of the inclusive decays of heavy quarkonium within an effective field theory framework is in terms of expectation values of the 4 -fermion operators of NRQCD [8]. At this respect our procedure can give a master equation which relates these matrix elements with wave-functions at the origen and some non-perturbative constants independent of the mass and the bound state dynamics. Given a four-fermion operator $O$, the master equation is the following $(|H\rangle$ represents a generic heavyquarkonium state at rest, $\mathbf{P}=0$ ):

$$
\begin{aligned}
& \langle H|O(\mathbf{0})| H\rangle=\frac{1}{\langle\mathbf{P}=0 \mid \mathbf{P}=0\rangle} \int d^{3} \mathbf{r} \int d^{3} \mathbf{r}^{\prime} \\
& \times \int d^{3} \mathbf{R} \int d^{3} \mathbf{R}^{\prime}\langle\mathbf{P}=0 \mid \mathbf{R}\rangle\langle n j l s \mid \mathbf{r}\rangle \\
& \times\left[\left\langle\underline{0} ; \mathbf{x}_{1} \mathbf{x}_{2}\left|\int d^{3} \xi O(\xi)\right| \underline{0} ; \mathbf{x}_{1}^{\prime} \mathbf{x}_{2}^{\prime}\right\rangle\right] \\
& \times\left\langle\mathbf{R}^{\prime} \mid \mathbf{P}=0\right\rangle\left\langle\mathbf{r}^{\prime} \mid n j l s\right\rangle,
\end{aligned}
$$

where $\mathbf{r}=\mathbf{x}_{1}-\mathbf{x}_{2}, \mathbf{r}^{\prime}=\mathbf{x}_{1}^{\prime}-\mathbf{x}_{2}^{\prime}, \mathbf{R}=\left(\mathbf{x}_{1}+\mathbf{x}_{2}\right) / 2$ and $\mathbf{R}^{\prime}=\left(\mathbf{x}_{1}^{\prime}+\mathbf{x}_{2}^{\prime}\right) / 2$ (note that $\left\langle\mathbf{R}^{\prime} \mid \mathbf{P}=0\right\rangle=1$ and $\left.\langle\mathbf{P}=0 \mid \mathbf{P}=0\rangle=\int d^{3} x\right)$. The state $\left|\underline{0} ; \mathbf{x}_{1} \mathbf{x}_{2}\right\rangle$ represents the heavy quark-antiquark ground state of the QCD Hamiltonian in the $1 / \mathrm{m}$ expansion (see Ref. [11] for details).

It is especially important that we can describe octet matrix elements, which were thought that could not be related with a Schrödinger-like formulation in any way. We have shown that this is not so. Nevertheless, we would like to stress that even for the singlet matrix elements, which were thought to be related with the wave-function at the origen of some Schrödinger-like formulation, our results are not trivial because no such formulation existed in the non-perturbative case before. We have provided it, formalizing the connection between NRQCD matrix elements and wave-functions computed in a Schrödinger equation and showing how this connection can be improved in a systematic way.
Our results allow to reduce significantly, by a factor of two, the number of non-perturbative constants compared with the number of NRQCD matrix elements if we assume that all the states below the $D-\bar{D}$ and $B-\bar{B}$ production threshold are in the dynamical situation $\Lambda_{Q C D} \gg m v^{2}$. In particular, one can think of combinations that are free of non-perturbative effects (see [11]).

I would like to thank N. Brambilla, D. Eiras, J. Soto and A. Vairo for collaboration on this topic.

\section{REFERENCES}

1. A. Pineda and J. Soto, Nucl. Phys. B (Proc. Suppl.) 64, 428 (1998); N. Brambilla, A. Pineda, J. Soto and A. Vairo, Nucl. Phys. B566, 275 (2000).

2. N. Brambilla, A. Pineda, J. Soto and A. Vairo, Phys. Rev. D63, 014023 (2001).

3. A. Pineda and A. Vairo, Phys. Rev. D63, 054007 (2001); E-ibid. D64, 039902 (2001).

4. K.G. Wilson, Phys. Rev. D10, 2445 (1974); L. Susskind, Les Houches lectures (1976).

5. E. Eichten and F.L. Feinberg, Phys. Rev. D23, 2724 (1981); M.E. Peskin, in Proc. 11th SLAC Institute, SLAC Report No. 207, 151 (1983); D. Gromes, Z. Phys. C26, 401 (1984).

6. A. Barchielli, E. Montaldi and G.M. Prosperi, Nucl. Phys. B296, 625 (1988); E-ibid. 303, 752 (1988); A. Barchielli, N. Brambilla and G. Prosperi, Nuovo Cimento 103A, 59 (1990).

7. A.P. Szczepaniak and E.S. Swanson, Phys. Rev. D55, 3987 (1997).

8. G.T. Bodwin, E. Braaten and G.P. Lepage, Phys. Rev. D51, 1125 (1995), E-ibid. D55, 5853 (1997).

9. Y. Chen, Y. Kuang and R.J. Oakes, Phys. Rev. D52, 264 (1995); G.S. Bali, K. Schilling and A. Wachter, Phys. Rev. D56, 2566 (1997); N. Brambilla and A. Vairo, Nucl. Phys. B (Proc. Suppl.) 74, 210 (1999).

10. R. Tafelmayer, Diploma Thesis, Heidelberg (1986).

11. N. Brambilla, D. Eiras, A. Pineda, J. Soto and A. Vairo, hep-ph/0208019.

12. N. Brambilla, D. Eiras, A. Pineda, J. Soto and A. Vairo, Phys. Rev. Lett. 88, 012003 (2002). 